\title{
Removal of carbon films by oxidation in narrow gaps: Thermo-oxidation and Plasma-Assisted studies
}

\author{
I. Tanarro ${ }^{1}$ J. A. Ferreira ${ }^{2}$, V. J. Herrero ${ }^{1}$, F. L. Tabarés ${ }^{2}$ and C. Gómez-Aleixandre ${ }^{3}$ \\ ${ }^{1}$ Instituto de Estructura de la Materia, CSIC, C/Serrano 123, 28006 Madrid, Spain \\ ${ }^{2}$ Laboratorio Nacional de Fusión, As. Euratom/Ciemat, Avda. Complutense 22, 28040 \\ Madrid, Spain \\ ${ }^{3}$ Instituto de Ciencia de Materiales de Madrid, CSIC, C/Sor Juana Inés de la Cruz 3, \\ Cantoblanco, 28049 Madrid, Spain
}

\begin{abstract}
The removal of hard amorphous hydrogenated carbon $(\mathrm{a}-\mathrm{C}: \mathrm{H})$ films from narrow gaps simulating the macro-brush structures present in controlled fusion devices has been investigated. Films with thickness of $50-150 \mathrm{~nm}$ were generated through plasma assisted chemical vapor deposition (PACVD) in glow discharges of $\mathrm{CH}_{4} / \mathrm{He}$ on $\mathrm{Si}$ and stainless steel plates. The deposited plates were then arranged to form sandwich structures building narrow gaps and were subject to erosion by exposure to $\mathrm{O}_{2} / \mathrm{He}$ plasmas and to thermal oxidation by $\mathrm{O}_{2}$ and by a $\mathrm{NO}_{2} / \mathrm{N}_{2}(1: 1)$ mixture. In the plasma etching experiments, the deposited layers were only partially removed by the plasma at the side wall gap surfaces, but were efficiently removed at the bottom of the gap. In the thermo-oxidation experiments, the deposited films were effectively and homogeneously removed with oxygen at $670 \mathrm{~K}$ and with the $\mathrm{NO}_{2} / \mathrm{N}_{2}$ mixture at $\mathrm{T}>570 \mathrm{~K}$.
\end{abstract}

PSI18 keywords: Amorphous Films, Carbon-based materials, Erosion \& Deposition, Wall conditioning.

JNM keywords: Coatings and Coated Particles, Heat Treatment, Plasma-Materials Interaction, Surface Effects.

PACS: 28.52.Fa, 52.40.Hf, 52.77.Bn, 68.60.Dv 


\section{Introduction}

The use of carbon materials in the high flux area of fusion devices, including the ITER divertor for the first phase, is seriously hampered by the formation of co-deposits of carbon and the plasma fuel which are found to migrate to remote areas not reached by the fusion plasma. This problem has triggered the development of a series of in-situ cleaning techniques aimed at the prevention or removal of these co-deposits or at least of their tritium content $[1,2]$. Among these, the high temperature oxidation [3],[4] and the glow discharge-driven erosion or isotope exchange have been considered for ITER operation. The Glow Discharge technique has already been tested in some fusion devices, with variable success [5]. On the other hand, the possibility of trapping of these co-deposits in gaps such as those existing between the tiles of the plasma facing components (PFC's) or within the castellated structure of the divertor modules poses an extra constrain to the available cleaning technique, even for the nominal PFC's [6]. In this work, the removal of carbon films from the gaps existing in castellated structures such as that of the macro brush design [7] has been addressed. For that purpose, several metallic structures with gaps widths between 0,25 and $1 \mathrm{~mm}$, and $25 \mathrm{~mm}$ depth, in which sides and bottom, a hard carbon-film layer has been deposited, were exposed to various cleaning conditions. Thermo-oxidation by oxygen and $\mathrm{NO}_{2} / \mathrm{N}_{2}$ mixtures and glow discharges in $\mathrm{He} / \mathrm{O}_{2}$ mixtures were tested. A model to simulate the results of plasma cleaning in gaps is also presented.

\section{Film deposition and simulation of gap structures}

Films of hard a-C: $\mathrm{H}$ characteristics were produced on stainless steel (SS) and silicon samples by PACVD in D.C. glow discharge reactors, similar to those described in [2],[8]. The geometry of the chamber was cylindrical (Ø $250 \mathrm{~mm}, 300 \mathrm{~mm}$ height) with a rod-like anode entering from a side of the reactor. Samples was grounded and directly immersed in the plasma. The films were generated from a mixture of $\mathrm{He}(80 \%)$ and $\mathrm{CH}_{4}(20 \%)$ at $1 \mathrm{~Pa}$. The plasma current was fixed at $100 \mathrm{~mA}$. A methane consumption of $\sim 30 \%$ and deposition rates of $\sim 0.1 \mathrm{~nm} \mathrm{~s}^{-1}$ were estimated from mass spectrometry. The film thickness was recorded in-situ by laser interferometry (at $670 \mathrm{~nm}$ ). Additionally, profilometry, colorimetry and ellipsometry [9] were used for ex-situ thickness measurement. The values obtained by in situ laser interferometry yield values between 100 and $150 \mathrm{~nm}$ depending on the point measured by the laser. The generated hard films had a typical $\mathrm{H} / \mathrm{C}$ ratio of 0,4 [10]. 
In order to simulate the constrained geometry of the gaps of the macro-brush structures, the a-C: $\mathrm{H}$ layers previously deposited on two separate plates at direct exposure to the methane plasmas were assembled to build the walls of flat box-like containers having only a slit for access (Fig. 1). The plates had typically a square basis of $\approx 25 \mathrm{~mm}$ side and $1 \mathrm{~mm}$ thickness. In the thermo-oxidation experiments, the open slit was $1 \mathrm{~mm}$ wide. In the case of plasma-assisted removal, entrance slits with widths of $0,25,0,4$ or $1 \mathrm{~mm}$ were employed. Both, the side walls and the bottom of the simulated gap structures were covered with the carbonaceous films at the start of the removal experiments. The film was deposited with the structure open (see figure 1), and the structure was closed under vacuum before the cleaning.

\section{Removal by plasma exposure}

A plasma composition of $20 \% \mathrm{O}_{2}$ and $80 \% \mathrm{He}(1 \mathrm{~Pa})$ was used for the erosion of the carbon layers measured by mass spectrometry. The plasma current was fixed at $100 \mathrm{~mA}$, corresponding to an averaged ion flux of $\approx 3.10^{14} \mathrm{~cm}^{-2} \mathrm{~s}^{-1}$. Langmuir probe measurements yielded electron densities of $\approx 5.10^{9} \mathrm{~cm}^{-3}$ and temperatures of $\approx 3 \mathrm{eV}$. A consumption of a $30 \%$ of injected $\mathrm{O}_{2}$ was measured by mass spectrometry. For the gas flow values used, this corresponds to atom fluxes about one order of magnitude larger than ion fluxes, assuming $100 \%$ losses at the walls. Erosion rates for direct exposed samples under these conditions [9] are $\sim 0,18 \mathrm{~nm} / \mathrm{s}$, so that complete cleaning of the external walls was achieved in less than $15 \mathrm{~min}$. For a better attack into the gap, $20 \mathrm{~min}$ exposure time was used. After exposure to the plasma, the film thickness profile along the covered wall was measured by ex-situ laser interferometry. Thickness profiles for the three values of the gap width used are shown in the upper panel of Fig. 2, together with the profile before the plasma erosion. These measurements were carried out on a silicon sample inserted in the gap, modifying the set-up shown in figure 1 . The samples were supported with a Teflon plug ( $5 \mathrm{~mm}$ length) bolted to the structure at the bottom of the gap (giving a non deposited surface that was used as reference). These steps represent the first $4 \mathrm{~mm}$ in figure 2. As this point was used as reference the top of the gap corresponds to $21 \mathrm{~mm}$ position.

Only the few uppermost mm of the covered plate (i. e. those closer to the plasma) are eroded, and the extension of the eroded area is larger for wider gaps, as expected. The spatial resolution of these measurements is limited by the laser spot size $(0.5 \mathrm{~mm})$. The structure observed in the depth profile is due to the shadows produced by the structure. At the bottom face of the gap, i.e. at the face opposite to the open entrance slit, $25 \mathrm{~mm}$ away from the plasma, full carbon removal was found (similar results were observed in previous 
works with a slit width of $3 \mathrm{~mm}$ [11]). In order to shed more light on this unexpected effect (due to the larger depth of the gap), an electrically isolated metallic ribbon was placed at the bottom of the gap during plasma exposure. Currents of a few $\mu \mathrm{A}$ and a floating potential of $\sim 30 \mathrm{~V}$ (as compared with the $400 \mathrm{~V}$ value of the plasma discharge) were recorded at this location. A further characterization of this effect is presently underway.

\section{Removal by thermal oxidation}

A cylindrical stainless steel oven connected to the vacuum chamber was used for thermal oxidation. The oxidizing gases were introduced to the oven when its temperature was already stabilized at the desired value.

The box-like samples placed inside the oven were exposed to successive one hour sessions of thermal oxidation in an atmosphere of 50 mbar of slowly flowing pure oxygen at 585,620 and $670 \mathrm{~K}$. The appearance of the samples hardly changed with the first two thermal oxidation processes at 585 and $620 \mathrm{~K}$, which indicates that the a-C: $\mathrm{H}$ films were very inefficiently removed at these temperatures. In contrast, after oxidation with $\mathrm{O}_{2}$ at 670 $\mathrm{K}$, the films were largely, although not totally, eliminated. In all cases, the effect of $\mathrm{O}_{2}$ on the films was the same in the internal and external walls of the box-like samples. The estimated average erosion rates for $\mathrm{O}_{2}$ at $670 \mathrm{~K}$ was $\sim 100 \mathrm{~nm} / \mathrm{h}$, a value consistent with previous literature data on the erosion of a-C:H films in $\mathrm{O}_{2}$ or air atmospheres at similar temperatures [4],[5].

Oxidation was also performed with a commercial $\mathrm{NO}_{2} / \mathrm{N}_{2}(1: 1)$ mixture. Three, one hour oxidation processes, were carried out at $50 \mathrm{mbar}$ and temperatures of 520, 570 and 620 $\mathrm{K}$. After oxidation at $520 \mathrm{~K}$, the films were not appreciably changed. At $570 \mathrm{~K}$, the films were largely removed both in the internal and external faces of the box-like sample structure. After oxidation at $620 \mathrm{~K}$, the samples were completely cleaned.

Time resolved mass spectrometric measurements were recorded during oxidation processes. When significant erosion was achieved (i. e. oxidation with $\mathrm{O}_{2}$ at $\mathrm{T}=670 \mathrm{~K}$ and with $\mathrm{NO}_{2}$ at $\mathrm{T} \geq 570 \mathrm{~K}$ ), an increase in the $\mathrm{CO}_{2}$ concentration was observed upon introduction of the oxidizing gas into the oven. After some minutes, the concentration of $\mathrm{CO}_{2}$ began to fall and levelled off slowly. Signals at masses $46\left(\mathrm{NO}_{2}\right)$ and $32\left(\mathrm{O}_{2}\right)$ grew with time and also tended to level off as oxidation reactions ceased in the oven. This tendency to equilibrium is shown in Fig. 3 for oxidation with $\mathrm{NO}_{2} / \mathrm{N}_{2}$ at $570 \mathrm{~K}$. Signals at $\mathrm{m} / \mathrm{q}=2,15$, 26, 27, 28 and 30 show a behaviour qualitatively similar to that of $\mathrm{CO}_{2}$. These masses correspond to $\mathrm{H}_{2}$, hydrocarbon fragments $\left(\mathrm{CH}_{\mathrm{x}}, \mathrm{C}_{2} \mathrm{H}_{\mathrm{y}}\right)$, and $\mathrm{CO}$ and $\mathrm{NO}$, also liberated during film erosion. An estimate of the $\mathrm{CO}$ and $\mathrm{NO}$ produced during oxidation is 
complicated because major contributions at masses 28 and 30 are due to $\mathrm{N}_{2}$, present in the feed mixture, and to $\mathrm{NO}$ fragmented from $\mathrm{NO}_{2}$ upon detection at the spectrometer.

\section{Surface characterization}

The surfaces with the deposited a-C:H layers were characterized after exposure to the cleaning medium by means of Glow Discharge-Optical Emission Spectroscopy (GD-OES) [12]. An Ar RF glow discharge is used to erode the surfaces of the plates and to excite the eroded material. A set of detectors tuned to different elements and arranged in a Rowland circle is used for emission analysis. Depth profiles can be determined after calibration with standard materials of known composition [12]. Film thickness values before oxidation deduced from the GD-OES data were in excellent agreement with the interferometry results mentioned above. One advantage of this method is that all kind of elements prone to excitation inside the plasma are identified.

Fig. 4 shows the atomic composition at the surface of a deposited Si plate after exposure to the $\mathrm{He} / \mathrm{O}_{2}$ plasma. The two panels correspond to two points from the inner face of the gap located at about 5 and $15 \mathrm{~mm}$ from the slit entrance, for a slit width of $0.25 \mathrm{~mm}$. Under these conditions no significant film erosion was observed (see Fig 2) and a $\mathrm{C} / \mathrm{H}$ layer was found at the surface. The layer closer to the entrance slit has a $20 \%$ proportion of $\mathrm{O}_{2}$ atoms. Deeper inside the gap, the amount of oxygen is much smaller. In neither case does the oxygen enter the bulk of the plate.

In the experiments of thermal oxidation leading to a significant surface cleaning (i.e. with $\mathrm{O}_{2}$ at $670 \mathrm{~K}$ and with $\mathrm{NO}_{2} / \mathrm{N}_{2}$ for $\mathrm{T} \geq 570 \mathrm{~K}$ ), the percentage of $\mathrm{O}$ atoms found at the surface was always high (close to $60 \%$ ), but the penetration of $\mathrm{O}$ atoms inside the bulk of the SS plate was also small. After oxidation in $\mathrm{O}_{2}$ at $670 \mathrm{~K}$, only a small amount of $\mathrm{C}$ atoms (always less than 15\%) was found at the surface. After oxidation in $\mathrm{NO}_{2}+\mathrm{N}_{2}$ at $570 \mathrm{~K}$, a percentage of $\mathrm{C}$ atoms up to $25 \%$ is found in a very thin layer at the least eroded areas (Fig. 5 ), whereas for $620 \mathrm{~K}$, the corresponding proportion of $\mathrm{C}$ atoms is about $7 \%$.

\section{Discussion}

The films produced in our plasmas can survive exposure to pure oxygen up to $\approx 670 \mathrm{~K}$. This temperature is significantly higher than that reported for Tokamak co-deposits [3] and therefore, these films represent an extreme case of what is to be found in real fusion experiments. In that sense, the fact that the $\mathrm{NO}_{2} / \mathrm{N}_{2}$ mixture is able to lower this temperature to $\sim 570 \mathrm{~K}$ is highly promising for future, low $\mathrm{T}$ applications, as in the case of ITER. With respect to the cleaning of gaps by $\mathrm{O}_{2} / \mathrm{He}$ plasmas, the present finding of a cleaning effect at 
the bottom of the gap, even for a depth of $25 \mathrm{~mm}$ is indeed surprising [12],[13]. Moreover, a current of few $\mu \mathrm{A}$ was recorded at this remote, hindered position. In contrast, plasma exposure proved inefficient for the removal of films at the walls of the gap. Film erosion by the plasma was found to be limited to the vicinity of the gap entrance and dependent on gap width. In order to gain more insight, the results of Fig.2 were fitted to a simple exponential decay for the first millimeters near the gap entrance. As shown in Fig. 6.a, simple exponential functions provide a good description of the measured data. Inverse characteristic decay lengths of $0,71,0,49$ and $0,2 \mathrm{~mm}^{-1}$ are obtained for the $0,25,0.4$ and 1 $\mathrm{mm}$ slits, respectively. Interestingly, these inverse characteristic lengths, $\lambda$, are to a good approximation inversely proportional to the respective slit widths, $\Delta$, so that $\lambda . d \sim 0,2$, thus suggesting pure geometrical effects. The decrease of the ion flux to the gap walls as a function of depth is much faster than that of the film erosion, as illustrated in Fig.6.b for a gap width of $1 \mathrm{~mm}$.

On the other hand, currents of $\sim 100 \mu \mathrm{A}$ would be required at the slit bottom in order to justify the full $\mathrm{C}$ removal observed, if a sputtering yields of $1 \mathrm{C}$ atom/ion is assumed [10]. This value is almost an order of magnitude higher than that experimentally recorded.

All the analysis made so far points to a strong contribution of non-ionic species to the cleaning mechanism, as suggested in previous works [13][14]. Neglecting the first 1-2 mm at the entrance, the propagation of the active species, A, into the gap can be evaluated from the continuity equation taking into account that the active species can only react at the walls and its concentration falls as it travels deeper in the gap:

$0=-\frac{1}{4} \Delta \cdot L \cdot \bar{v} d n-2 \cdot \frac{1}{4} n \cdot L \cdot S \cdot \bar{v} d h$

$n=n_{0} \exp \left(-\frac{2 S}{\Delta} h\right)=n=n_{0} \exp (-b h)$

Where $\Delta$ stands for the gap width, $n$ for density (of the active species), $h$ for the distance from the slit into the gap, L for its length, $v$ is assumed to be the thermalized velocity of the species and $\mathrm{S}$ its surface loss probability. In the equation 1, first term accounts for the flux of active species coming in the gap, and the second term accounts for the losses in the walls, considering a one dimensional model. Eq. 2 reproduces the two main features of the experimental findings, the exponential decay with $h$ (with a characteristic decay length, $\lambda=1 / b$ ), and the constant value of $\Delta \cdot b$. From eq. 2, a value of $S=0,1$ is then inferred, corresponding to a small reactivity of the active species in the absence of ions, as postulated elsewhere [10]. From the flux of atomic oxygen to the open surfaces and the experimental erosion rates, a ratio of $0,6 \mathrm{C} / \mathrm{O}$ atom in the presence of ion bombardment is deduced. Note 
that for a low $\mathrm{S}$ value in a metal/C mixed scenario, such as expected for the $\mathrm{Be}$ wall in ITER, the available concentration of atoms impinging of the carbon surfaces will be governed by the surface loss probability in the metallic areas, which could eventually lead to higher cleaning rates than those observed in laboratory experiments.

Thermal oxidation, especially with $\mathrm{NO}_{2}$ at $\mathrm{T}>570 \mathrm{~K}$ offers interesting perspectives for the cleaning of carbon deposits in the macro-brush structures of fusion devices. The mass spectra recorded during film removal indicate that in addition to the expected products of the oxidation reactions like $\mathrm{CO}_{2}, \mathrm{CO}$ and $\mathrm{NO}$, small hydrocarbons and $\mathrm{H}_{2}$ are also liberated, but more work is needed for the elucidation of the film erosion mechanisms.

\section{Summary and Conclusions}

The removal by plasma etching in $\mathrm{O}_{2} / \mathrm{He}$ and $\mathrm{N}_{2} / \mathrm{H}_{2}$ mixtures and by thermo-oxidation at 50 mbar in $\mathrm{O}_{2}$ and $\mathrm{N}_{2} / \mathrm{NO}_{2}$, of hard a-C:H films $(\mathrm{H} / \mathrm{C} \sim 0.4)$ produced by PACVD from $\mathrm{CH}_{4} / \mathrm{He}$ mixtures, has been investigated at several temperatures and for constrained geometries, simulating the macro-brush structures present in controlled fusion devices. The main findings are now summarized.

Thermo-oxidation in $\mathrm{O}_{2}$ during 1 hour removes $\mathrm{a} \approx 100 \mathrm{~nm}$ film only for exposure temperatures of $\sim 670 \mathrm{~K}$. This value should be an upper limit for high quality films, and it is expected [3] that real Tokamak co-deposits will show more relaxed removal conditions. Replacing molecular oxygen by a $\mathrm{NO}_{2} / \mathrm{N}_{2}$ mixture (1:1) produces higher erosion rates. At $570 \mathrm{~K}$, the effect is similar to that of $670 \mathrm{~K}$ in $\mathrm{O}_{2}$.

Plasma oxidation in an $\mathrm{O}_{2} / \mathrm{He}$ mixture removes very efficiently the films at open surfaces $(0,18 \mathrm{~nm} / \mathrm{s})$, however, its penetration into thin gaps $(0,25,0,4$ and $1 \mathrm{~mm}$ wide) is very limited and the deposited films inside the gap walls are only slightly eroded. In contrast to the gap walls, a complete removal of the deposits at the bottom of the gap, 25 $\mathrm{mm}$ deep, was found.

With the help of a simple model, a surface loss probability of 0,1 for the active species at the inner gap walls was deduced. This low probability might be related with a smaller reactivity of the neutral active species in the absence of ions, which are geometrically screened to a large extent inside the gap.

\section{Acknowledgements}


We thank R. Serna for the ellipsometry measurements. This work has been funded by EFDA under the Technical Task, TW6-TPP-Gapox; and by the MEC of Spain under grants FIS2007-61686 and ENE2006-14577-CO4-CO3/FTN.

\section{References}

[1] G. Federici et al, Nucl.Fus. 41(2001) 1967-2137

[2] F. L. Tabarés, et al, Plasma Phys. Control. Fusion 44 (2002) L37.

[3] J.W. Davis and A.A.Haasz. Phys.Scripta T91 (2001) 33

[4] W. Wang, W. Jacob, J. Roth, J. Nucl. Matter. 245 (1997) 66

[5] C. Hopf et al., J Nucl Mater.363-365 (2007) 882 and references therein.

[6] M. Rubel, these proceedings.

[7] G. Janeschitz et al. J,Nucl. Matter. 290-293 (2001) 1

[8] I. Tanarro, et al, J. Phys. Chem. A 111 (2007) 9003

[9] R. Serna et al , J. Appl. Phys. 84 (1998) 4509

[10] B. Landkammer, A. von Keudell and W. Jacob. J. Nucl. Matter. 264-264 (1999) 48

[11] J. A. Ferreira, F.L. Tabarés and D. Tafalla, J. Vac. Sci.Technol. A25 (2007) 746

[12] R. Escobar Galindo et al, Spectrochimica Acta Part B 61 (2006) 545

[13] J. A. Ferreira, F. L. Tabarés, D. Tafalla, J Nucl Mater.363-365 (2007) 888

[14] W.L.Hsu. J. Vac.Sci.Technol. A7 (1989) 1047 


\section{Figure Captions}

Fig. 1. Scheme of the plates used for deposition and removal of a-C: $\mathrm{H}$ films.

Fig. 2. Film thickness profile before and after its exposure to cleaning plasmas for different gap widths.

Fig. 3. Time resolved mass spectra during oxidation with $\mathrm{NO}_{2} / \mathrm{N}_{2}$ at $570 \mathrm{~K}$, after background subtraction and normalization by the total pressure.

Fig. 4. GD-OES measurements of relative atom concentrations at the surface of an a-C: $\mathrm{H}$ covered Si plate, after exposure to $\mathrm{He} / \mathrm{O}_{2}$ plasmas through a $0.25 \mathrm{~mm}$ slit. (A) $5 \mathrm{~mm}$ from the gap entrance. (B) $15 \mathrm{~mm}$ from the gap entrance.

Fig. 5. GD-OES relative atom concentrations in SS plates after thermal oxidation in $\mathrm{NO}_{2} / \mathrm{N}_{2}$ at $570 \mathrm{~K}$, inside a gap $1 \mathrm{~mm}$ wide.

Fig. 6. (A) Fitting of the eroded thickness data from Fig.2 to simple exponential decays for 3 gap widths. (B) Expected ion flux to the inside walls of a gap $1 \mathrm{~mm}$ wide as a function of the distance from the plasma (dashed line). Experimental erosion profile (solid line). 
Figures of "Removal of carbon films by oxidation in narrow gaps: Thermo-oxidation and Plasma-Assisted studies" by I. Tanarro et al

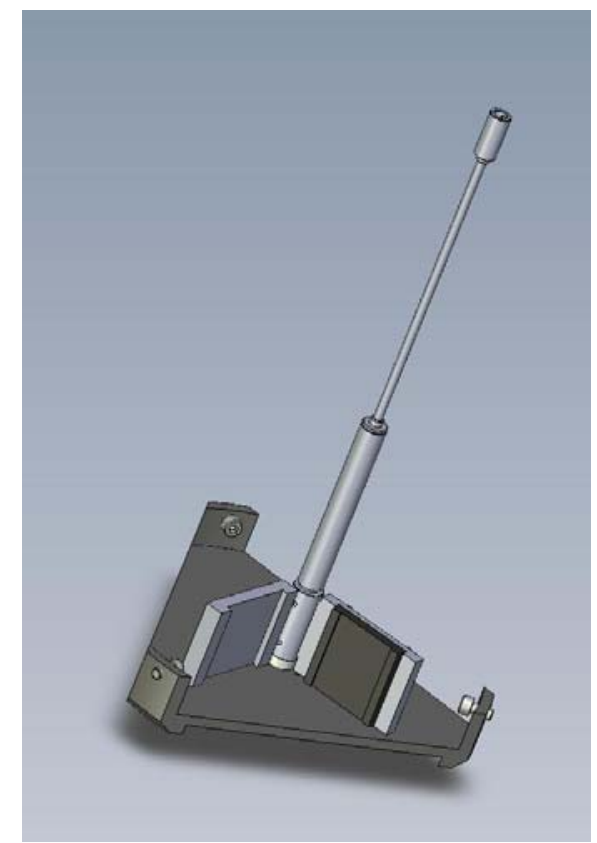

Figure 1

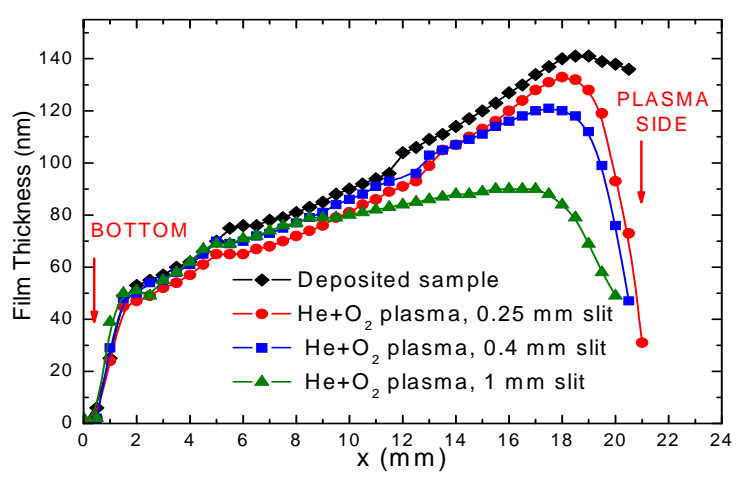

Figure 2

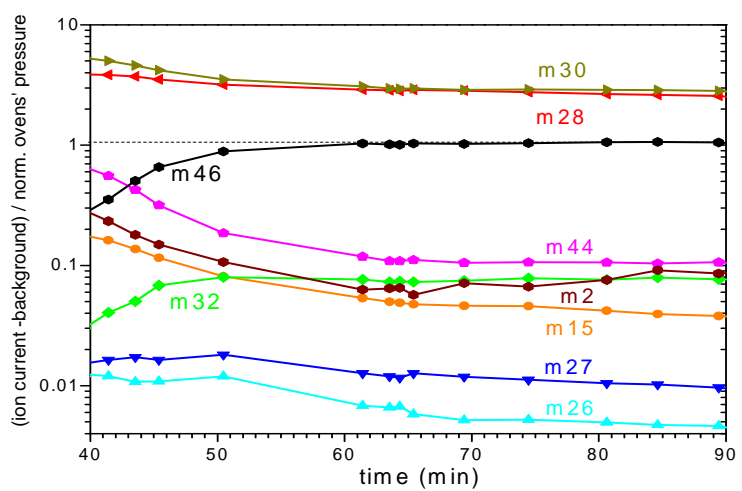

Figure 3 

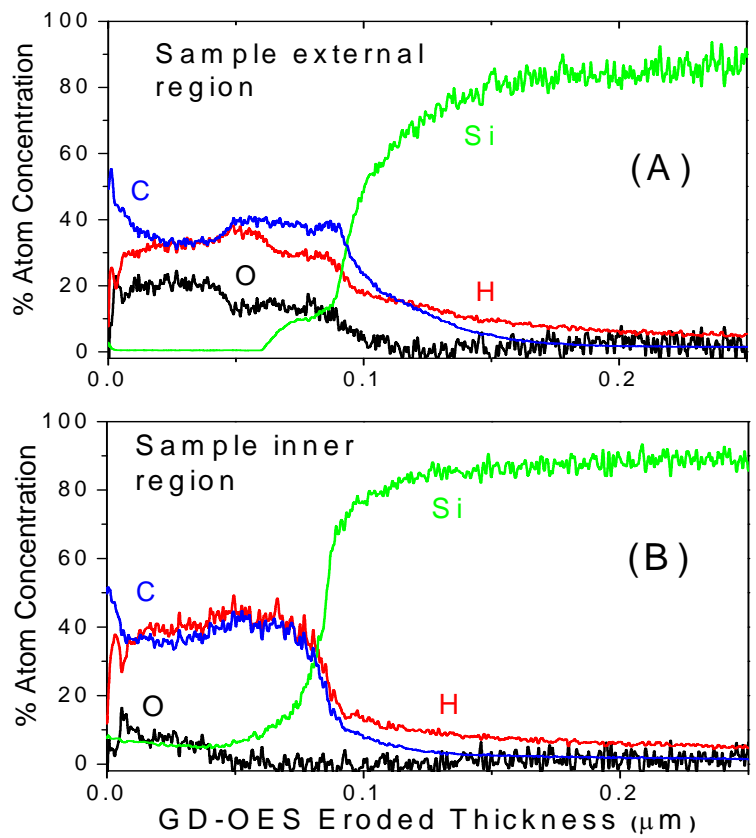

Figure 4

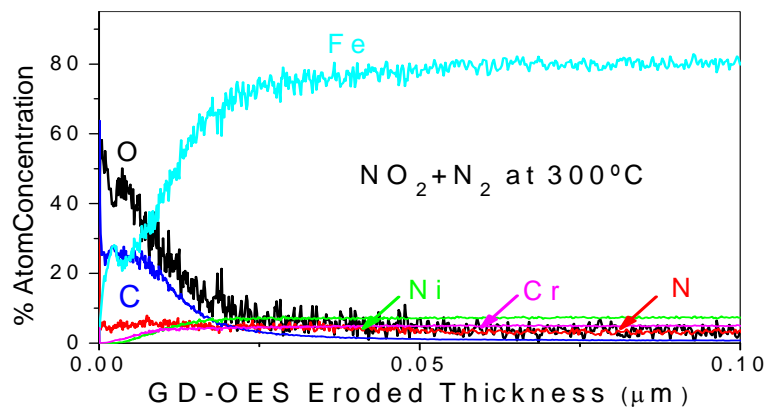

Figure 5

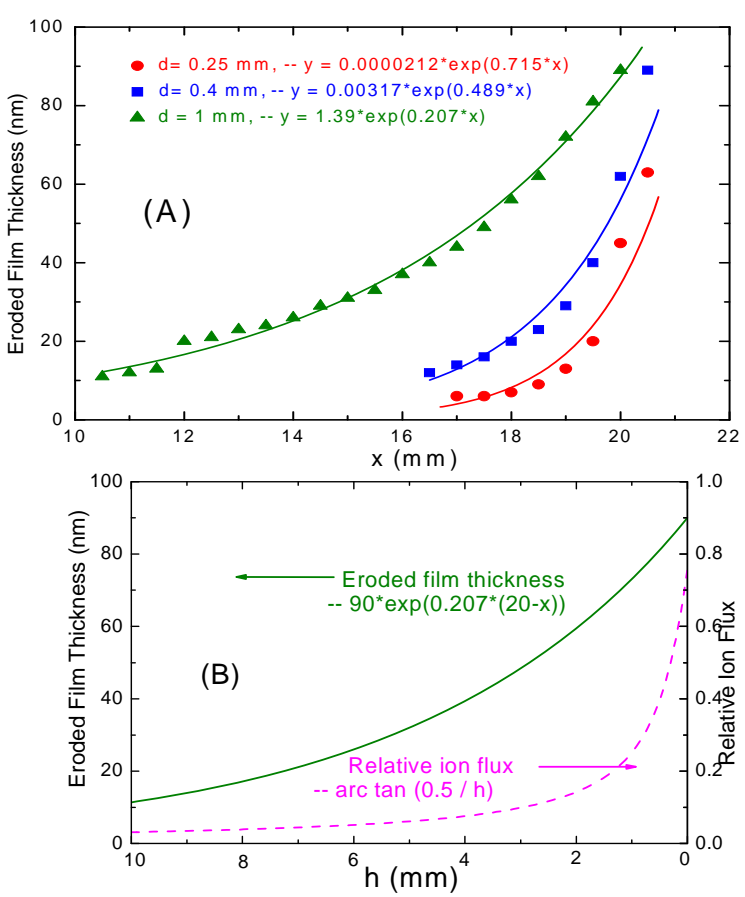

Figure 6 